\title{
Some Results of Best Proximity Point in Regular Cone Metric Spaces
}

\author{
M. Chinaie, N. Rajaee and M. Ahmadi Baseri*
}

(Communicated by Nihal YILMAZ ÖZGÜR)

\begin{abstract}
The purpose of this paper is to provide sufficient conditions for the existence of a best proximity point for various types of cyclic contraction maps. Our results extend and improve certain recent results in the literature.
\end{abstract}

Keywords: Best proximity point; cyclic contraction map; cone L-function; regular cone metric.

AMS Subject Classification (2010): Primary: 41A65; Secondary: 41A52; 46N10.

${ }^{*}$ Corresponding author

\section{Introduction and preliminaries}

Let $A$ and $B$ nonempty subsets of a metric space $X$. If there is a pair $\left(x_{0}, y_{0}\right) \in A \times B$ for which $d\left(x_{0}, y_{0}\right)=$ $d(A, B)$, that

$$
d(A, B)=\inf \{d(x, y): x \in A, y \in B\},
$$

then the pair $\left(x_{0}, y_{0}\right)$ is called a best proximity pair for $A$ and $B$. We can find the best proximity pair of the sets $A$ and $B$, by considering a map $T: A \cup B \rightarrow A \cup B$ such that $T(A) \subseteq B$ and $T(B) \subseteq A$. The point $x \in A \cup B$ is a best proximity point for $T$ if, $d(x, T x)=d(A, B)$. A map $T: A \cup B \rightarrow A \cup B, T(A) \subseteq B, T(B) \subseteq A$ is called cyclic contraction [3] if, for some $k \in(0,1)$ the condition

$$
d(T x, T y) \leq k d(x, y)+(1-k) d(A, B)
$$

holds for all $x \in A, y \in B$.

In 2003, Kirk et al. proved fixed point results for cyclic contraction maps [8]. In 2006, Eldered and Veeramani obtained best proximity point results for cyclic contraction maps [3].

Theorem 1.1. [3] Let $A$ and $B$ be nonempty closed subsets of a complete metric space $X$. Let $T: A \cup B \rightarrow A \cup B$ be a cyclic contraction map. Let $x_{0} \in A$ and define $x_{n+1}=T x_{n}$. Suppose $\left\{x_{2 n}\right\}$ has a convergent subsequence in $A$. Then there exists $x \in A$ such that $d(x, T x)=d(A, B)$.

Best proximity point theory of cyclic contraction maps has been studied by many authors see [1, 3, 9] and references therein. In 2007, Huang and Zhang [6] introduced cone metric spaces as a generalization of metric spaces. Then in [10] some results about characterization of best approximations in the cone metric spaces are studied. In 2011, Haghi et al [4] obtained best proximity points for cyclic contraction maps on regular cone metric spaces. In 2012, Karapinar [7], obtained best proximity point for certain cyclic contraction maps in metric spaces. In 2013, Amini and et al [2], introduce a new class of cyclic generalized contraction maps and it is shown that the best proximity point property for closed and convex subsets of a uniformly convex Banach space holds.

Received : 10-January-2015, Accepted : 15-January-2016 
In this paper, we obtain some existence of best proximity point theorems for various types of cyclic contraction maps, which are the generalization of some results in the literature. To prove our results in the next section we recall some definitions and facts. In the present paper $E$ stands for a real Banach space. A subset $P$ of $E$ is called a cone if and only if

(i) $P$ is closed, nonempty and $P \neq\{0\}$;

(ii) $a, b \in \mathbb{R}^{+}$and $x, y \in P$ implies $a x+b y \in P$;

(iii) $x \in P$ and $-x \in P$ implies $x=0$.

We define a partial ordering $\leq$ with respect to $P$ by $x \leq y$ if and only if $y-x \in P . x<y$ will stand for $x \leq y$ and $x \neq y$, while $x \ll y$ will stand for $y-x \in$ int $P$, where int $P$ denotes the interior of $P$.

A map $f: P \rightarrow P$ is said to be increasing (strictly increasing) whenever $x \leq y$ implies that $f(x) \leq f(y)(x<y$ implies that $f(x)<f(y))$.

A cone $P$ is said to be normal if there is a number $M>0$ such that for all $x, y \in E$

$$
0 \leq x \leq y \quad \text { implies } \quad\|x\| \leq M\|y\| .
$$

The least positive number $M$ satisfying the above inequality is called the normal constant of cone $P$. [6]

Definition 1.1. [12] A nonempty subset $A$ of $(X, d)$, is said to be bounded above if there exists $c \in$ int $P$ such that $c-d(x, y) \in P$ for all $x, y \in A$.

The cone $P$ is called regular if every increasing sequence which is bounded from above is convergent. That is, if $\left\{x_{n}\right\}_{n \geq 1}$ is a sequence such that $x_{1} \leq x_{2} \leq \ldots \leq y$ for some $y \in E$, then there is $x \in E$ such that $\lim _{n \rightarrow \infty} \|$ $x_{n}-x \|=0$. Equivalently, the cone $P$ is regular if and only if, every decreasing sequence which is bounded from below is convergent.

Lemma 1.1. [11] Every regular cone is normal.

Definition 1.2. [6] Let $X$ be a nonempty set. Suppose that a mapping $d: X \times X \rightarrow E$ satisfies:

(d1) $0 \leq d(x, y)$ for every $x, y \in X$ and $d(x, y)=0$ if and only if $x=y$;

(d2) $d(x, y)=d(y, x)$ for every $x, y \in X$;

(d3) $d(x, y) \leq d(x, z)+d(z, y)$ for every $x, y, z \in X$.

Then $d$ is called a cone metric and $(X, d)$ is called a cone metric space.

Example 1.1. [5] Let $E=\left(L^{1}[0,1],\|\cdot\|_{1}\right), P=\{f \in E: f \geq 0 \quad$ a.e. $\},(X, \rho)$ be a metric space and $d: X \times X \rightarrow E$ defined by $d(x, y)=f_{x, y}$, where $f_{x, y}(t)=\rho(x, y) t^{2}$. Then $(X, d)$ is a regular cone metric space. In fact, if $\left\{f_{n}\right\}_{n \geq 1}$ is an increasing sequence and there is $g \in L^{1}$ such that $f_{1} \leq f_{2} \leq \ldots \leq f_{n} \leq \ldots \leq g$ for almost every where $x$, then $\left\{f_{n}\right\}_{n \geq 1}$ converges to a function $f$ a.e. on $X$. Then, $f_{n} \leq f \leq g$ (a.e.) for all $n \geq 1$. Thus $g-f_{1} \in L^{1}, g-f_{n} \leq g-f_{1}$ for all $n \geq 1$ and $\lim _{n \rightarrow \infty} g-f_{n}=g-f$ (a.e.)

Hence by the Lebesgue dominated convergence theorem, $f \in L^{1}$ and

$\lim _{n \rightarrow \infty}\left\|f_{n}-f\right\|_{1}=0$. So, the cone $P$ is regular.

Definition 1.3. [4] Let $A$ and $B$ nonempty subsets of cone metric space $(X, d)$. An element $p \in P$ is said to be a lower bound for $A \times B$ whenever

$$
p \leq d(a, b),
$$

for all $(a, b) \in A \times B$. If $p \geq q$ for all lower bound $q$ for $A \times B$, then $p$ is called the greatest lower bound for $A \times B$. We denote it by $d(A, B)$.

Clearly, $d(A, B)$ is a unique vector in $P$.

Definition 1.4. [4] A map $\psi: P \rightarrow P$ is called cone L- function whenever $\psi(0)=0, \psi(s)>0$ for all $s \in P$ with $s \neq 0$ and there exists $\delta_{s} \gg 0$ such that $\psi(t) \leq s$ for all $s \leq t \leq s+\delta_{s}$.

Lemma 1.2. [4] Let $\psi: P \rightarrow P$ be a cone L-function and $\left\{s_{n}\right\}$ a decreacing sequence in $P$ such that $s_{n+1}<\psi\left(s_{n}\right)$ for all $n \geq 1$. Then $\lim _{n \rightarrow \infty} s_{n}=0$. 


\section{Main results}

Throughout this section, $E$ is a normed space, $(X, d)$ is regular cone metric space, $\leq$ is the partial ordering with respect to $P$ and $A, B$ are nonempty subsets of $X$.

Theorem 2.1. Let $T: A \cup B \rightarrow A \cup B$ be a map such that $T(A) \subseteq B$,

$T(B) \subseteq A$ and

$$
\begin{aligned}
d(T x, T y) & \leq k \max \{d(x, y),(1 / 2)\{d(T x, x)+d(T y, y)\}\} \\
& +(1-k) d(a, b)
\end{aligned}
$$

for all $(a, b),(x, y) \in A \times B$, for some $k \in(0,1)$. Then, $d(A, B)$ exists.

Proof. The $\max \{d(x, y),(1 / 2)\{d(T x, x)+d(T y, y)\}\}=d(x, y)$ is known (see [4]). Let

$$
\max \{d(x, y),(1 / 2)\{d(T x, x)+d(T y, y)\}\}=(1 / 2)\{d(T x, x)+d(T y, y)\} .
$$

Take $x_{0} \in A \cup B$, set $x_{n+1}=T x_{n}$ and $d_{n+1}=d\left(x_{n+1}, x_{n}\right)$ for all $n \geq 1$. Then

$$
d_{n+1} \leq(k / 2)\left\{d_{n+1}+d_{n}\right\}+(1-k) d(a, b),
$$

for all $(a, b) \in A \times B$, which is equivalent to

$$
d_{n+1} \leq \frac{k / 2}{1-k / 2} d_{n}+\frac{1-k}{1-k / 2} d(a, b)
$$

for each $(a, b)$ in $A \times B$. It follows that $d_{n+1} \leq d_{n}$ for all $n \geq 1$. By the regularity of the cone $P$, there exists $p \in P$ such that $d_{n} \rightarrow p$ as $n \rightarrow \infty$. Thus $p \leq d(a, b)$ holds for any $(a, b)$ in $A \times B$. Now if $q$ is a lower bound for $A \times B$, then $q \leq d_{n}$ for all $n \geq 1$, and so, $q \leq p$. Therefore, $d(A, B)=p$.

Note that, inequality (2.1) is equivalent to

$$
d(T x, T y) \leq k \max \{d(x, y),(1 / 2)\{d(T x, x)+d(T y, y)\}\}+(1-k) d(A, B),
$$

in metric spaces.

Theorem 2.2. Suppose that the conditions of Theorem 2.1 hold, $x_{0} \in A$ and $x_{n+1}=T x_{n}$ for all $n \geq 1$. If $\left\{x_{2 n}\right\}$ has a convergent subsequence in $A$, then there exists $x \in A$ such that $d(x, T x)=d(A, B)$.

Proof. Let $\left\{x_{2 n_{k}}\right\}$ be the convergent subsequence of $\left\{x_{2 n}\right\}$ in $A$ with $x_{2 n_{k}} \rightarrow x \in A$. Since

$$
p=d(A, B) \leq d\left(x, x_{2 n_{k}-1}\right) \leq d\left(x, x_{2 n_{k}}\right)+d\left(x_{2 n_{k}}, x_{2 n_{k}-1}\right),
$$

for each $k \geq 1,\left\{d\left(x_{2 n_{k}}, x_{2 n_{k}-1}\right)\right\}$ is a subsequence of $\left\{d_{n}\right\}$, hence $d\left(x, x_{2 n_{k}-1}\right) \rightarrow p$ as $n \rightarrow \infty$. As

$$
p \leq d\left(T x, x_{2 n_{k}}\right) \leq d\left(x, x_{2 n_{k}-1}\right),
$$

for all $k \geq 1$. It follows that $d(x, T x)=p=d(A, B)$.

Theorem 2.3. Let $T: A \cup B \rightarrow A \cup B$ be a map such that $T(A) \subseteq B, T(B) \subseteq A$ and

$$
d(T x, T y) \leq a d(x, y)+b\{d(T x, x)+d(T y, y)\}+c d(a, b),
$$

for all $(a, b),(x, y) \in A \times B$, where $a, b, c$ are constant such that $a, b, c \geq 0$ and $a+2 b+c<1$. Then $d(A, B)$ exists.

Proof. Take $x_{0} \in A \cup B$. Set $x_{n+1}=T x_{n}$ and $d_{n+1}=d\left(x_{n+1}, x_{n}\right)$ for all $n \geq 1$. Then

$$
d_{n+1} \leq a d_{n}+b\left\{d_{n+1}+d_{n}\right\}+c d(a, b),
$$

for all $(a, b) \in A \times B$. So

$$
d_{n+1} \leq \frac{a+b}{1-b} d_{n}+\frac{c}{1-b} d(a, b)
$$

for each $(a, b) \in A \times B$. So

$$
d_{n+1} \leq k d_{n}+(1-k) d(a, b)
$$

for all $(a, b)$ in $A \times B$, where $k=(a+b) /(1-b)$. Hence, $d_{n+1} \leq d_{n}$ for all $n \geq 1$. Similar to the prove of Theorem 2.1 we obtain the result. 
Note that, inequality (2.2) is equivalent to

$$
d(T x, T y) \leq a d(x, y)+b\{d(T x, x)+d(T y, y)\}+c d(A, B),
$$

in metric spaces.

Theorem 2.4. Suppose that the conditions of Theorem 2.3 hold, $x_{0} \in A$ and $x_{n+1}=T x_{n}$ for all $n \geq 1$. If $\left\{x_{2 n}\right\}$ has a convergent subsequence in $A$. Then there exists $x \in A$ such that $d(x, T x)=d(A, B)$.

Proof. The proof is similar to the proof of Theorem 2.2.

Now, we will consider the best proximity points for a pair of mapping $(S, T)$, such that $S, T: A \cup B \rightarrow$ $A \cup B, S(A) \subseteq B$ and $T(B) \subseteq A$.

Theorem 2.5. Let $S, T: A \cup B \rightarrow A \cup B$ such that $S(A) \subseteq B, T(B) \subseteq A$ and

$$
d(S x, T y) \leq k d(x, y)+(1-k) d(a, b),
$$

for all $(a, b),(x, y) \in A \times B$, for some $k \in(0,1)$. Then, $d(A, B)$ exists.

Proof. Take $x_{0} \in A$, then $S x_{0} \in B$, so there exists $y_{0} \in B$ such that $y_{0}=S x_{0}$. Now $T y_{0} \in A$, so there exists $x_{1} \in A$ such that $x_{1}=T y_{0}$. Inductively, we define sequence $\left\{x_{n}\right\}$ and $\left\{y_{n}\right\}$ in $A$ and $B$, respectively by

$$
x_{n+1}=T y_{n}, \quad y_{n}=S x_{n} .
$$

Set $d_{n}=d\left(x_{n}, S x_{n}\right)$. Since

$$
\begin{aligned}
d_{n+1} & \leq k d\left(y_{n}, x_{n+1}\right)+(1-k) d(a, b) \\
& \leq k^{2} d_{n}+\left(1-k^{2}\right) d(a, b),
\end{aligned}
$$

for all $(a, b)$ in $A \times B$. It follows that $d_{n+1} \leq d_{n}$. Similar to the prove of Theorem 2.1 we obtain the result.

Note that, inequality (2.3) is equivalent to

$$
d(S x, T y) \leq k d(x, y)+(1-k) d(A, B)
$$

in metric spaces. Also, in case $S=T$, Theorem 2.5 reduce to the Theorem 2.1 in [4].

Theorem 2.6. Suppose that the conditions of Theorem 2.5 hold and the sequence $\left\{x_{n}\right\}$ and $\left\{y_{n}\right\}$ are generated by (2.4) for some $x_{0} \in A \cup B$. If both $\left\{x_{n}\right\}$ and $\left\{y_{n}\right\}$ have a convergent subsequence in $A$ and $B$ respectively, then there exist $x \in A$ and $y \in B$ such that

$$
d(x, S x)=d(A, B)=d(y, T y) .
$$

Proof. Set $d_{n}=d\left(x_{n}, S x_{n}\right)$. Let $\left\{y_{n_{k}}\right\}$ be a subsequence of $\left\{y_{n}\right\}$ such that $y_{n_{k}} \rightarrow y$. The relation

$$
p=d(A, B) \leq d\left(T y_{n_{k}}, y\right) \leq d\left(y_{n_{k}}, y\right)+d\left(y_{n_{k}}, T y_{n_{k}}\right)
$$

holds for each $k \geq 1$. Since

$$
d\left(y_{n_{k}}, T y_{n_{k}}\right) \leq k d_{n_{k}}+(1-k) d(a, b),
$$

for all $(a, b) \in A \times B$. It follows that $d\left(y_{n_{k}}, T y_{n_{k}}\right) \leq d_{n_{k}}$. Since $\left\{d\left(S x_{n_{k}}, x_{n_{k}}\right)\right\}$ is a subsequence of $\left\{d_{n}\right\}$, hence $\lim _{k \rightarrow \infty} d\left(S x_{n_{k}}, x_{n_{k}}\right)=p$. Thus

$$
\lim _{k \rightarrow \infty} d\left(y_{n_{k}}, T y_{n_{k}}\right)=p
$$

So $d\left(T y_{n_{k}}, y\right) \rightarrow p$ as $k \rightarrow \infty$. Now, for each $k \geq 1$

$$
\begin{aligned}
d\left(T y, y_{n_{k}}\right) & \leq k d\left(y, x_{n_{k}}\right)+(1-k) d(a, b) \\
& \leq k\left\{d\left(y, y_{n_{k}}\right)+d\left(y_{n_{k}}, x_{n_{k}}\right)\right\}+(1-k) d(a, b) .
\end{aligned}
$$


i.e.

$$
p=d(A, B) \leq d\left(T y, y_{n_{k}}\right) \leq k\left\{d\left(y, y_{n_{k}}\right)+d_{n_{k}}\right\}+(1-k) d(a, b),
$$

for all $(a, b) \in A \times B$. Letting $k \rightarrow \infty$, we have $d(T y, y)=p=d(A, B)$.

Similarly, it can be proved that $d(x, S x)=d(A, B)$.

In the following Theorem, the distance of $A$ and $B$ is obtained by considering the pair mapping $(S, T)$ in a regular cone metric space.

Theorem 2.7. Let $\psi: P \rightarrow P$ be a cone L-function. $S, T: A \cup B \rightarrow A \cup B$ such that $T(B) \subseteq A, S(A) \subseteq B$ and

$$
d(S x, T y)-p<\psi(d(x, y)-p),
$$

for all $(x, y) \in A \times B$ with $p<d(x, y)$, where $p$ is lower bounded for $A \times B$. Then $d(A, B)=p$.

Proof. Let $\left\{x_{n}\right\}$ and $\left\{y_{n}\right\}$ be as follows $x_{n+1}=T y_{n}, S x_{n}=y_{n+1}$ for some $\left(x_{0}, y_{0}\right) \in A \times B, n \in \mathbb{N}$. Also let $d_{n+1}=d\left(x_{n+1}, y_{n+1}\right)$, we have

$$
d_{n+1}-p<\psi\left(d_{n}-p\right) \leq d_{n}-p .
$$

By the regularity of the cone $P$, we have $d_{n+1} \leq d_{n}$. Hence, there exists $q \in P$ such that $\lim _{n \rightarrow \infty} d_{n}=q$. Then $p \leq q$. Put $s_{n}=d_{n}-p$. Since, $s_{n}>0$, we have $s_{n+1}<\psi\left(s_{n}\right) \leq s_{n}$. By Lemma 1.8, $\lim _{n \rightarrow \infty} s_{n}=0$. Thus, $\lim _{n \rightarrow \infty} d_{n}=p$ and so $d(A, B)=p=q$.

Note that, in case $S=T$. Theorem 2.7 reduce to the Theorem 2.4 in [4].

\section{Acknowledgment}

This research was partially supported by Center of Excellence for Mathematics (University of Isfahan).

\section{References}

[1] Al-Thagafi, M. A., Shahzad, N., Convergence and existence result for best proximity points. Nonliner Anal. 70 (2009), no.10, 3665-3671.

[2] Amini-Harandi, A., Hussain, N., Akbar, F., Best proximity point results for generalized contractions in metric spaces. Fixed Point Theory Appl. (2013), 2013:164, 13 pp.

[3] Eldred, A. A., Veeramani, P., Existence and convergence of best proximity points. J. Math. Anal. Appl. 323 (2006), no.2, 1001-1006.

[4] Haghi, R. H., Rakoc̃ević, V., Rezapour, Sh., Shahzad, N., Best proximity result in regular cone metric space. Rend. Circ. Mat. Palermo (2) 60 (2011), no.3, 323-327.

[5] Haghi, R. H., Rezapour, Sh., Fixed points of multifunctions on regular cone metric space. Expo. Math. 28 (2010), no.1, 71-77.

[6] Huang, L. G., Zhang, X., Cone metric spaces and fixed point theorems of contractive mappings. J. Math. Anal. Appl. 332 (2007), no.2, 1468-1476.

[7] Karapınar, E., Best proximity points of cyclic mappings. Appl. Math. Lett. 25 (2012), no.11, 1761-1766.

[8] Kirk, W. A., Sirinavasan, P. S., Veeramani, P., Fixed points for mappings satisfying cyclical contractive conditions. Fixed Point Theory 4 (2003), no.1, 79 - 89.

[9] Mohsenalhosseini, S. A. M, Mazaheri, H., Dehghan, M. A., Approximate best proximity pairs in metric space. Abstr. Appl. Anal. (2011), Art. ID 596971, 9 pp.

[10] Rezapour, Sh., Best approximations in cone metric spaces. Math. Morav. 11 (2007), 85-88. 
[11] Rezapour, Sh., Hamlbarani Haghi, R., Some notes on the paper "Cone metric spaces and fixed point theorems of contractive mappings". J. Math. Anal. Appl. 345 (2008), no.2, 719-724.

[12] Turkoglu, D., Abuloha, M., Cone metric spases and fixed point theorems in diametrically contractive mappings. Acta Mathematica Sinica, English Series. 26 (2010), no.3, 489-496.

\section{Affiliations}

M. ChINAIE

ADDRESS: Esfahan University, Faculty of Mathematics, Esfahan-Iran.

E-MAIL: m.chinaei@yahoo.com

N. RAJAEE

ADDRESS: Esfahan University, Faculty of Mathematics, Esfahan-Iran.

E-MAIL: nfs.rajaee@gmail.com

M. AHMADi BASERI

ADDRESS: Yazd University, Faculty of Mathematics, Yazd-Iran.

E-MAIL: marziehahmadi634@gmail.com 